\title{
Antiplatelet effects of ticagrelor versus clopidogrel after coronary artery bypass graft surgery: A single-center randomized controlled trial
}

Fei Xu, MD, ${ }^{\mathrm{a}}$ Wei Feng, MD, ${ }^{\mathrm{a}}$ Zhou Zhou, MD, ${ }^{\mathrm{b}}$ Yang Zhang, MD, ${ }^{\mathrm{b}}$ Xiaolin Diao, MS, Shengshou $\mathrm{Hu}, \mathrm{MD}^{\mathrm{a}}$ and Zhe Zheng, $\mathrm{MD}^{\mathrm{a}}$

\section{ABSTRACT}

Objective: The study objective was to compare the onset of platelet inhibition (inhibition of platelet aggregation) between ticagrelor $90 \mathrm{mg}$ twice per day and clopidogrel $75 \mathrm{mg}$ once per day in patients receiving coronary artery bypass grafting.

Methods: In a single-center, randomized, open-label study, 140 patients receiving coronary artery bypass grafting were randomly assigned to the aspirin + ticagrelor group or the aspirin + clopidogrel group in a 1:1 ratio. Participants in the aspirin + ticagrelor group took aspirin $100 \mathrm{mg}$ once per day and ticagrelor $90 \mathrm{mg}$ twice per day. Participants in the aspirin + clopidogrel group took aspirin $100 \mathrm{mg}$ once per day and clopidogrel $75 \mathrm{mg}$ once per day. Platelet function was determined before study treatment ( 0 hours); at 2 hours, 8 hours, 24 hours, and 72 hours after medication; and during follow-up at 30 days after surgery.

Results: Inhibition of platelet aggregation at 2 hours after the first drug administration was greater for the aspirin + ticagrelor group than for the aspirin + clopidogrel group (34.2\% [interquartile range, 9.1-66.0] vs $5.3 \%$ [interquartile range, $-14.3-22.0$ ], $P<.001)$ and at all times in the study period $(P<.001)$. More patients reached inhibition of platelet aggregation maximum within 24 hours in the aspirin + ticagrelor group than in the aspirin + clopidogrel group $(52.9 \%$ vs $27.5 \%, P=.006)$. The average inhibition of platelet aggregation maximum from 2 to 24 hours was still greater in the aspirin + ticagrelor group than in the aspirin + clopidogrel group $(72.3 \% \pm 15.4 \%$ vs $49.2 \% \pm 46.8 \%, P<.001)$. There were no differences in terms of bleeding or major adverse cardiac events between the 2 groups.

Conclusions: In patients receiving coronary artery bypass grafting, the onset of action was faster and the peak inhibition of platelet aggregation was higher with ticagrelor than with clopidogrel. (J Thorac Cardiovasc Surg 2019;158:430-7)

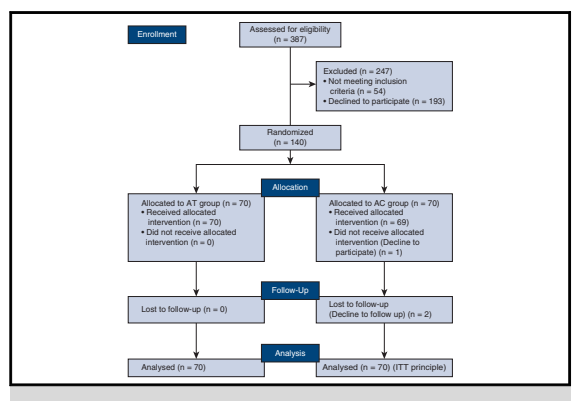

The trial flow diagram.

\section{Central Message}

In this randomized controlled trial of patients who underwent CABG, the onset of action was faster with ticagrelor than with clopidogrel, whereas the medication safety within 1 month was similar.

\section{Perspective}

Antiplatelet therapy is important for secondary prevention of CABG. However, despite the established comprehensive clinical use and benefit in patients undergoing $\mathrm{CABG}, \mathrm{AC}$ therapy has limitations, including the failure to elicit a complete antiplatelet response. Ticagrelor is a promising antiplatelet agent, but its efficacy in patients undergoing CABG has been studied less in the literature.

See Commentaries on pages 438 and 440
From the ${ }^{\mathrm{a}}$ Department of Cardiovascular Surgery, Fuwai Hospital, ${ }^{\mathrm{b}} \mathrm{Center}$ of Laboratory Medicine, Beijing Key Laboratory for Molecular Disease, Fuwai Hospital, and ${ }^{\mathrm{c}}$ Biostatistical Unit, Fuwai Hospital, Chinese Academy of Medical Sciences, and Peking Union Medical College, Beijing, China.

This study was funded by AstraZeneca (China) Co, Ltd

Clinical trial registry number. The trial was registered on www.Clinicaltrials.gov (Registry Number NCT02330640). Institutional Review Board approval: The study was approved by the ethics committee of Fuwai Hospital. Approval date: December 17, 2014. Approval number: 2014-599.

Received for publication Jan 18, 2018; revisions received Sept 30, 2018; accepted for publication Oct 2, 2018; available ahead of print Nov 27, 2018.

Address for reprints: Zhe Zheng, MD, Department of Cardiovascular Surgery, Fuwai Hospital, Chinese Academy of Medical Sciences, No. 167 North Lishi Rd, Xicheng District, Beijing, China (E-mail: zhengzhe@fuwai.com).

$0022-5223 / \$ 36.00$

Copyright $(c) 2018$ by The American Association for Thoracic Surgery

https://doi.org/10.1016/j.jtcvs.2018.10.032
Early graft occlusion is one of the major risk factors that negatively affects prognosis after coronary artery bypass grafting (CABG). Many factors, such as surgical trauma, cardiopulmonary bypass, and initial injury, contribute to platelet activation, which subsequently influences graft patency. ${ }^{1,2}$ Currently, early application of aspirin plus

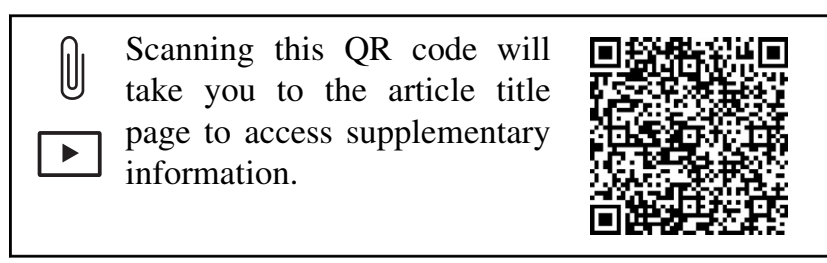




$$
\begin{aligned}
& \text { Abbreviations and Acronyms } \\
& \begin{aligned}
\text { ADP } & =\text { adenosine diphosphate } \\
\text { AT } & =\text { aspirin }+ \text { ticagrelor } \\
\text { AC } & =\text { aspirin }+ \text { clopidogrel } \\
\text { BARC } & =\text { Bleeding Academic Research } \\
& \text { Consortium } \\
\text { CABG } & =\text { coronary artery bypass grafting } \\
\text { DAPT } & =\text { dual antiplatelet therapy } \\
\text { IPA } & =\text { inhibition of platelet aggregation } \\
\text { ITT } & =\text { intention-to-treat } \\
\text { MACE } & \text { major adverse cardiovascular events } \\
\text { MI } & =\text { myocardial infarction } \\
\text { PRI } & =\text { platelet reactivity index } \\
\text { VASP-P }= & \text { vasodilator-stimulated phosphoprotein } \\
& \text { phosphorylation }
\end{aligned}
\end{aligned}
$$

P2Y12 receptor inhibitor, known as dual antiplatelet therapy (DAPT), after surgery has become a standardized prevention strategy for patients undergoing $\mathrm{CABG} .{ }^{3,4}$

DAPT, defined as medication of aspirin in combination with another antiplatelet drug, often consists of aspirin and clopidogrel, the effects of which were approved by a few studies. ${ }^{5-7}$ However, with more evidence coming out, it was found that the antiplatelet effect of clopidogrel varies significantly, and clopidogrel resistance was seen in approximately $30 \%$ of patients ${ }^{8,9}$; thus, clopidogrel may not provide satisfactory antiplatelet effects in the early postoperative period.

Different from clopidogrel, which requires liver enzyme to be activated, ticagrelor is characterized by rapid onset of action and high inhibition of platelet aggregation (IPA), resulting in obvious pharmacokinetic advantages over clopidogrel. ${ }^{10}$ However, in patients receiving CABG who develop intense inflammatory reaction and activated platelet function postoperatively, ${ }^{11,12}$ it is unknown whether ticagrelor performs better than clopidogrel. We hypothesized that ticagrelor is not inferior to clopidogrel in terms of antiplatelet effects for patients receiving CABG. Therefore, we conducted a randomized controlled trial to compare pharmacokinetics and safety aspects between ticagrelor and clopidogrel at the early stage after CABG.

\section{MATERIALS AND METHODS \\ Study Design}

This study was an open-label, randomized, controlled clinical trial. Patients were randomly assigned to the aspirin + ticagrelor (AT) group or the aspirin + clopidogrel (AC) group in a 1:1 ratio immediately after CABG, according to the random table generated by SAS software (SAS Institute Inc, Cary, NC). All the subjects were numbered, and each subject was assigned a random number according to a random number table. The subjects were ordered according to the random number they received. The first 70 subjects were assigned to the AT group, and the last 70 subjects were assigned to the AC group. The randomization was blinded to patients but not to operators. The study was approved by the ethics committee of Fuwai Hospital. The trial was registered on Clinicaltrials.gov (Registry Number NCT02330640). All participants gave written informed consent.

Between January 2016 and October 2016, we randomized 140 patients who underwent CABG. Patients were eligible if they met all of the following criteria: (1) treated by 2 designated surgeons who agreed to participate in this trial; (2) age 18 to 80 years; and (3) isolated CABG for the first time. Patients were not eligible if they had any 1 of the following conditions: (1) combined valvular surgery; (2) a second CABG; (3) emergency CABG, including selective CABG that transferred to emergency CABG; (4) serum creatinine greater than $130 \mu \mathrm{mol} / \mathrm{L}$; (5) oral clopidogrel therapy within 5 days before the CABG; (6) oral anticoagulation therapy (warfarin) that cannot be withheld; (7) history of gastrointestinal, vaginal bleeding, postoperative gastrointestinal bleeding, or cerebral hemorrhage; (8) active pathological bleeding (eg, active gastroduodenal ulcer or cerebral hemorrhage); (9) uric acid nephropathy; (10) any other condition that may influence platelet count and function; (11) contraindication to aspirin, clopidogrel, or ticagrelor; (12) participants of other trials within 30 days before the CABG; or (13) plan of pregnancy within 12 months. The procedure on the patients will be stopped if any of the conditions occurred after the CABG procedure: (1) postoperative chest drainage greater than $200 \mathrm{~mL} / \mathrm{h}$ for 2 hours or more; (2) need for reoperation for bleeding with cardiac tamponade; or (3) treated with intra-aortic balloon pump or extracorporeal membrane oxygenation after operation.

\section{Data Collection}

All the data were collected by trained clinical research staff, including research nurses or residents at Fuwai Hospital. The Case Report Forms were double entered into the database by trained technicians. Two auditors abstracted a random sample of $5 \%$ to $10 \%$ of medical records. The data submitted were compared with information in the medical records. When disagreement occurred, a supervisor adjudicated the variable to determine the final value.

As part of the current study protocol, all participants discharged alive were required to return for an outpatient follow-up visit at 1 month after surgery. For those who had reported any adverse events during the 1month period, their medical records in the outpatient clinic were further reviewed for confirmation. For any major clinical events that were reportedly treated in other hospitals, patients were asked to give a copy of all relevant medical records by mail. Overall, the completion rate was $100 \%$.

\section{Procedures}

Except study treatment, all randomized patients received the standard CABG protocol. Participants started study treatments after extubation. Participants in the AT group took aspirin $100 \mathrm{mg}$ daily and ticagrelor $90 \mathrm{mg}$ twice daily. Participants in the AC group took aspirin $100 \mathrm{mg}$ daily and clopidogrel $75 \mathrm{mg}$ daily. Because of the risk of bleeding from surgery, we did not give participants a loading dose.

\section{Surgical Procedures}

All surgeries were performed by 2 senior surgeons, with the same anesthetists and perfusionists. Standard techniques were adopted. In general, during off-pump CABG surgery, several cardiac positioning techniques and coronary artery stabilizers were adopted as previously described. ${ }^{13}$ On-pump CABG was performed with standard cardiopulmonary bypass techniques, using cold antegrade and retrograde blood cardioplegia, and moderate systemic hypothermia $\left(30^{\circ} \mathrm{C}-34^{\circ} \mathrm{C}\right)$.

\section{Outcome Measures}

Because the onset of both agents was 2 hours after administration, the primary end point was defended as IPA at 2 hours after the first dose of study treatment. IPA was calculated as follows: PA is platelet aggregation, 
$\mathrm{b}$ is pre-dosing, and $\mathrm{t}$ is postdosing: IPA $\%=((\mathrm{PAb}-\mathrm{PAt}) / \mathrm{PAb}) \times 100 \%$. Platelet aggregation was measured by light-transmittance aggregometry at 2 hours in CABG recipients after the first dose of study drug. Secondary efficacy end points were the inhibition of platelet function measured by light-transmittance aggregometry at 0 hours, 8 hours, 24 hours, 3 days, and 30 days after the first dose of study drug; and the platelet reactivity index (PRI) at 0 hours, 2 hours, 8 hours, 24 hours, 3 days, and 30 days.

Safety measures included bleeding events according to the Bleeding Academic Research Consortium (BARC) definition (Table E1) ${ }^{14}$ and major adverse cardiovascular events (MACE). Considering the specificity of operation, the BARC type 1 or 2 bleeding events were not recorded in the early period of postoperation (7 days). MACE was a combination of all-cause mortality, myocardial infarction (MI), and repeat revascularization.

\section{Blood Sample Collection and Platelet Function Test}

Blood samples were collected from subjects for platelet function tests before study treatment ( 0 hours); at 2 hours, 8 hours, 24 hours, and 72 hours after medication; and during follow-up at 30 days after surgery. Two tubes of blood, $3 \mathrm{~mL}$ in each tube, were collected from peripheral veins; then, $109 \mathrm{mmol} / \mathrm{L}$ sodium citrate anticoagulant was added, giving a blood volume and anticoagulant ratio of 9:1. Two methods, namely, turbidimetry and measurement of vasodilator-stimulated phosphoprotein phosphorylation, were used to determine platelet function.

1. Turbidimetry. LBY-NJ4A Automated Platelet Aggregation Analyzer manufactured by Beijing Techlink Biomedical (Beijing, China) was used for analysis in this study. In principle, platelet aggregation rate was determined by turbidimetry. Adenosine diphosphate (ADP) $20 \mu \mathrm{mol} / \mathrm{L}$ was added to platelet-rich plasma as an inducer to determine maximum platelet aggregation rate within 300 seconds.

2. Vasodilator-stimulated phosphoprotein phosphoryla-

tion assay. The VASP phosphorylation test was performed after blood collection by an experienced technician using Platelet VASP kits (Biocytex Co Ltd, Marseille, France) according to the instruction manual. Briefly, blood samples were mixed in vitro with ADP or prostaglandin E1. Each blood sample was incubated with a 16C2FITC antibody, followed by a goat anti-mouse fluorescence staining in isothiocyanate polyclonal reagent. Enzyme-linked immunosorbent assay was used to determine vasodilatorstimulated phosphoprotein phosphorylation on serine 239 of platelets in fresh whole blood. When prostaglandin E1 alone or together with ADP was added to the samples, optical density obtained at $450 \mathrm{~nm}$ was used to calculate PRI

\section{STATISTICS \\ Determination of Sample Size}

The trial was designed to detect at least a $15 \%$ difference in the IPA at 2 hours between the 2 groups. We conservatively assumed the standard deviation of IPA was $30 \%$. With a 2 -sided .05 significance level and a $10 \%$ dropout rate, we calculated that 140 patients $(70$ patients in each group) would yield $80 \%$ power to detect that ticagrelor was superior to clopidogrel.

\section{Statistical Analysis}

The analysis was based on an intention-to-treat analysis that included patients who were randomized to a treatment group and contributed baseline data, regardless of whether patients received at least 1 dose of study drug or not. For the demographics and surgical characteristics, summary statistics were constructed by using frequencies and proportions for categoric data and mean and standard deviation for continuous variables. The baseline variables were compared by $t$ test or Wilcoxon rank-sum test for continuous variables or chi-square test or Fisher exact test for categoric variables, as appropriate.

For the primary analysis comparing the treatment effects, we did the test for normality, $t$ test, and Wilcoxon rank-sum test to compare IPA at 2 hours between the groups (AT vs AC group). Without imputing missing observations for the primary analysis, the mixed-effects model for repeated measures was applied as a sensitivity analysis to examine the effect of missing data. To estimate global variation between groups, we fitted the mixed-effects model including fixed effects for treatment group, hour, and treatment group-by-hour interaction with random coefficients for the patient. According to the trend of change, we separately fitted 2 similar models with IPA values at 2, 8, and 24 hours, and at 24,72 , and 720 hours to obtain the slopes of onset and stable period. The multiple comparisons of IPA, within and between groups at each time from 2 to 720 hours, were performed using multiple test with Bonferroni-adjusted correction. The IPAmax from 2 to 720 hours and the time to reach to IPAmax were computed and compared between groups.

The analyses for the PRI were performed in the same manner as the IPA analysis except for computing PRImin and time to reach to PRImin, and chi-square test or Fisher exact test was conducted for comparing bleeding events and MACE between groups. Sensitivity analysis was conducted by excluding the 3 patients ( 2 patients declined follow-up, and 1 patient did not received the allocated treatment).

All statistical testing was 2 sided, at a significance level of .05, and all analyses used SAS version 9.4 (SAS Institute Inc) and estimated the mixed models and analysis of variance using the GLM procedure in SAS.

\section{RESULTS \\ Demographics and Baseline Characteristics}

From January 2016 to October 2016, 387 patients were evaluated for eligibility and 140 patients were randomized. One patient did not take the study treatment, and 2 patients were lost to follow-up (Figure 1). According to the principle of intention-to-treat analysis, all patient data are recorded and analyzed. Of the 140 patients randomized (70 in the AT group and 70 in the AC group), there was a good balance between the 2 groups with respect to baseline characteristics (Table 1). Mean age of patients was 59.5 years $( \pm 9.2)$, and $24.8 \%$ were female. Four patients had Canadian Cardiovascular Society (CCS) class IV, and 18.2\% of patients had New York Heart Association (NYHA) class III or IV. The preoperative platelet counts were not significantly different between the 2 groups. Some $81.4 \%$ of patients received off-pump $\mathrm{CABG}$, and the graft numbers were not significantly different between the AT and AC groups (Table 1). 


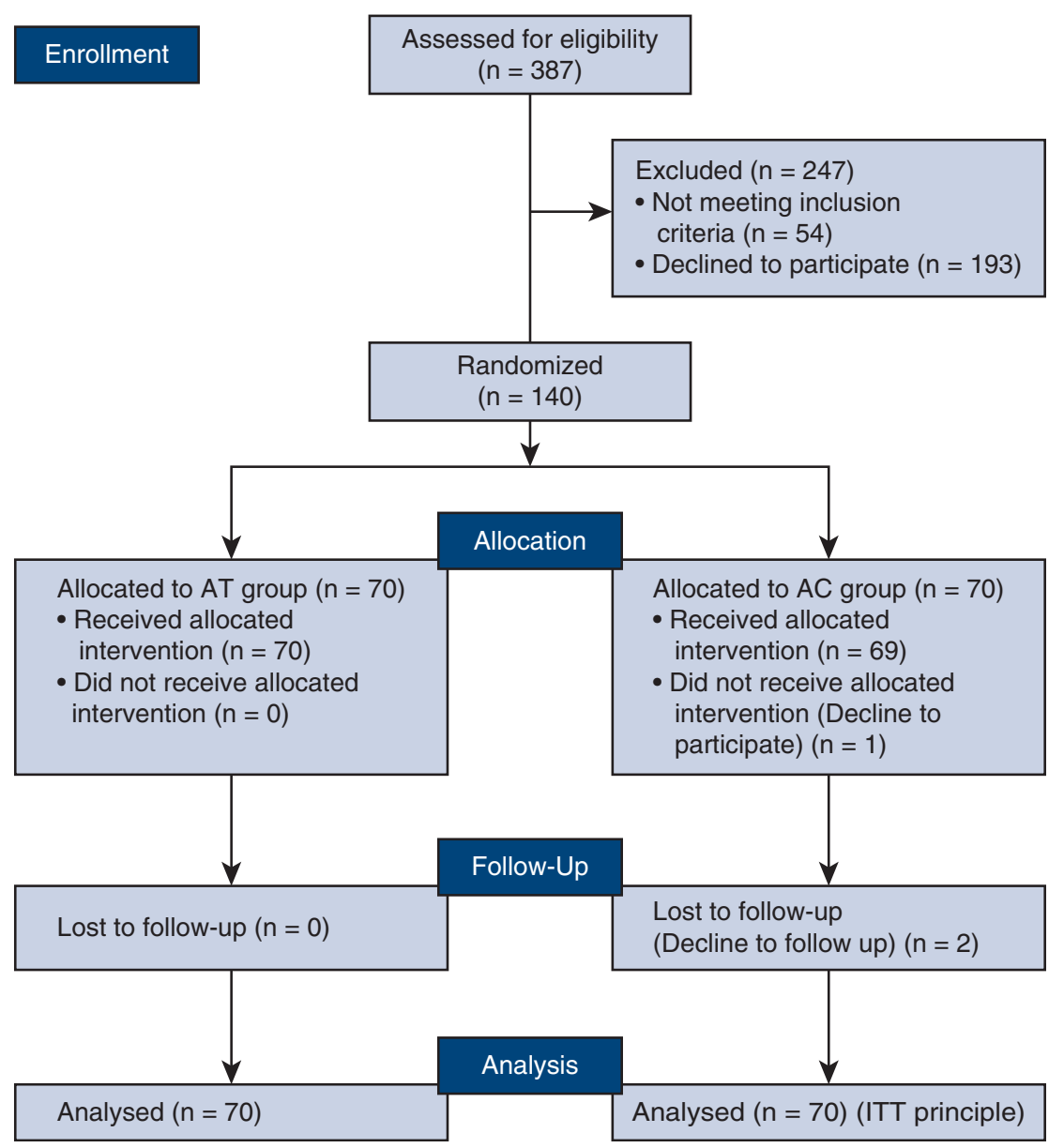

FIGURE 1. Study flow diagram. Flow chart of the study, including the number of screened patients, patients who withdrew, and patients who were in the final analysis. During the study period, 140 patients were eligible for this study. After 3 patients quit, we randomized the 137 patients to 2 groups: AT group $(\mathrm{n}=70)$ and $\mathrm{AC}$ group $(\mathrm{n}=67)$. IPA at 2 hours after the first dose of study treatment was the primary end point. AT, Aspirin + ticagrelor; $A C$, aspirin + clopidogrel; ITT, intention-to-treat.

\section{Platelet Function}

Overall, the 2 groups responded equally to aspirin. The primary end point for onset, IPA at 2 hours after the first drug administration, was greater for AT than for AC (median, $34.2 \%$; interquartile range, $9.1-66.0$ vs $5.3 \%$; interquartile range, $-14.3-22.0, P<.001$; Table $2 ; P<.01$ for the normality test) and at all times in the study period $(P<.001$; Figure $2, A)$. In the AT group, IPA did not differ between 24 and 72 hours, and between 72 and 720 hours after first administration, whereas in the AC group, IPA was greater at 720 hours than at 72 hours $(P<.001$; Video 1$)$.

As shown in Figure 2, $B$, more patients reached IPAmax within 24 hours in the AT group than in the AC group $(52.9 \%$ vs $27.5 \%, P=.002)$; the percentages of patients reaching IPAmax between 24 and 72 hours were not different between the 2 groups (AT vs AC: $17.1 \%$ vs $18.8 \%, P=.825$ ); however, between 72 and 720 hours, more patients in the AC group reached IPAmax when compared with those in the AT group (AT vs AC: $30.0 \%$ vs $53.6 \%, P=.006)$. The rate of onset slope of the antiplatelet effect curve as assessed by IPA from 2 to 24 hours after the first drug administration was greater in the AT group than in the AC group $(0.9 \pm 0.2$ vs $0.5 \pm 0.3 \mathrm{IPA} /$ h, $P<.001)$.

As is shown in Figure $3, A$ and $B$, more patients reached PRImin within 24 hours in the AT group than in the AC group $(48.6 \%$ vs $18.6 \%, P<.001)$; the percentages of patients reaching PRImin between 24 and 72 hours were not different between the 2 groups (AT vs AC: $31.4 \%$ vs $20.0 \%, P=.122$ ); however, between 72 and 720 hours, more patients in the AC group reached PRImin when compared with those in the AT group (AT vs AC: $20.0 \%$ vs $61.4 \%, P<.001$ ). The slope from 0 to 24 hours was less in the AT group than in the AC group $(-0.019 \pm 0.001$ vs $-0.003 \pm 0.002 \mathrm{PRI} / \mathrm{h}, P<.001)$.

From 2 to 24 hours, both IPAmax and PRI were greater in the AT group than in the AC group: The median IPAmax 
TABLE 1. Demographics, baseline characteristics, and surgical data

\begin{tabular}{|c|c|c|}
\hline & AT $(n=70)$ & $\mathbf{A C}(n=70)$ \\
\hline \multicolumn{3}{|l|}{ Baseline characteristics } \\
\hline Age (y) (mean \pm SD) & $58.6 \pm 8.9$ & $60.3 \pm 7.5$ \\
\hline \multicolumn{3}{|l|}{ Gender } \\
\hline Male & $77.1 \%, 54$ & $72.9 \%, 51$ \\
\hline Female & $22.9 \%, 16$ & $27.1 \%, 19$ \\
\hline Weight & $72.8 \pm 11.1$ & $71.8 \pm 9.9$ \\
\hline Hypertension & $68.6 \%, 48$ & $62.9 \%, 44$ \\
\hline Hyperlipidemia & $67.1 \%, 47$ & $67.1 \%, 47$ \\
\hline Diabetes mellitus & $67.1 \%, 47$ & $67.1 \%, 47$ \\
\hline Previous AMI & $35.7 \%, 25$ & $40.0 \%, 28$ \\
\hline PCI history & $14.3 \%, 10$ & $11.4 \%, 8$ \\
\hline $\mathrm{EF}(\%)$ & $58.4 \pm 10.6$ & $59.4 \pm 8.6$ \\
\hline LVEDD (mm) & $50.2 \pm 6.3$ & $49.5 \pm 5.3$ \\
\hline \multicolumn{3}{|l|}{ CCS class } \\
\hline 1 & $10.0 \%, 7$ & $11.4 \%, 8$ \\
\hline 2 & $42.9 \%, 30$ & $45.7 \%, 32$ \\
\hline 3 & $30.0 \%, 21$ & $25.7 \%, 18$ \\
\hline 4 & $17.1 \%, 12$ & $17.1 \%, 22$ \\
\hline \multicolumn{3}{|l|}{ NYHA } \\
\hline $\mathrm{I}(\%)$ & $7.1 \%, 5$ & $12.9 \%, 9$ \\
\hline II ( $\%)$ & $72.9 \%, 51$ & $70.0 \%, 49$ \\
\hline III $(\%)$ & $17.1 \%, 12$ & $15.7 \%, 11$ \\
\hline IV $(\%)$ & $2.9 \%, 2$ & $1.4 \%, 1$ \\
\hline Hemoglobin (g/L) & $136.9 \pm 14.5$ & $132.6 \pm 19.0$ \\
\hline Platelet $\left(* 10^{9} / \mathrm{L}\right)$ & $213.8 \pm 64.3$ & $211.3 \pm 59.4$ \\
\hline Prothrombin time (s) & $14.5 \pm 13.3$ & $14.6 \pm 13.4$ \\
\hline Creatinine (mmol/L) & $82.5 \pm 15.5$ & $83.8 \pm 16.7$ \\
\hline \multicolumn{3}{|l|}{ Preoperative medication } \\
\hline Aspirin & $58.6 \%, 41$ & $54.2 \%, 38$ \\
\hline$\beta$-blocker & $100 \%, 70$ & $100 \%, 70$ \\
\hline ACEI/ARB & $32.9 \%, 23$ & $30 \%, 21$ \\
\hline Calcium antagonist & $51.4 \%, 36$ & $61.4 \%, 43$ \\
\hline Statins & $71.4 \%, 50$ & $68.6 \%, 48$ \\
\hline Nitrates & $88.6 \%, 62$ & $92.3 \%, 65$ \\
\hline euroSCORE II & $1.2 \pm 0.5$ & $1.2 \pm 0.7$ \\
\hline STS score & $0.7 \pm 0.6$ & $0.8 \pm 0.6$ \\
\hline \multicolumn{3}{|l|}{ Surgical data } \\
\hline \multicolumn{3}{|l|}{ Surgical procedure } \\
\hline On-pump & $17.1 \%, 12$ & $20 \%, 14$ \\
\hline Off-pump & $82.9 \%, 58$ & $80 \%, 56$ \\
\hline \multicolumn{3}{|l|}{ No. of grafts } \\
\hline 1 & $1.4 \%, 1$ & $0 \%, 0$ \\
\hline 2 & $8.6 \%, 6$ & $7.1 \%, 5$ \\
\hline 3 & $35.7 \%, 25$ & $25.7 \%, 18$ \\
\hline 4 & $45.7 \%, 32$ & $58.6 \%, 41$ \\
\hline 5 & $8.6 \%, 6$ & $7.1 \%, 5$ \\
\hline 6 & $0.0 \%, 0$ & $1.4 \%, 1$ \\
\hline
\end{tabular}

Values are mean $\pm \mathrm{SD}$ or $\mathrm{n}(\%) . A T$, Aspirin + ticagrelor; $A C$, aspirin + clopidogrel; $S D$, standard deviation; $A M I$, acute myocardial infarction; $P C I$, percutaneous coronary intervention; $E F$, ejection fraction; $L V E D D$, left ventricular end-diastolic diameter; CCS, Canadian Cardiovascular Society; NYHA, New York Heart Association; $A C E I / A R B$, angiotensin-converting enzyme inhibitor/angiotensin receptor blocker; euroSCORE, European System for Cardiac Operative Risk Evaluation; STS, Society of Thoracic Surgeons. from 2 to 24 hours was still greater in the AT group than in the AC group $(72.3 \% \pm 15.4 \%$ vs $49.2 \% \pm 46.8 \%$, $P<.001)$; however, the average time to reach to IPAmax from 2 to 720 hours was faster in the AT group than in the AC group $(235.8 \pm 320.0$ hours vs $403.8 \pm 343.1$ hours, $P<.0016$ ).

The same results were observed with PRI examinations. The median PRImin from 0 to 24 hours was less in the AT group than in the AC group $(0.1 \pm 0.1$ vs $0.8 \pm 0.2, P<.001)$; the average time to reach to PRImin from 0 to 720 hours was faster in the AT group than in the AC group $(174.7 \pm 275.8$ hours vs $457.7 \pm 334.1$ hours, $P<.001)$.

\section{Clinical Outcomes}

No life-threatening bleeding (BARC type 4 and 5) was noted in the study period, and minor bleedings were not significantly different between the AT and AC groups: for type 1, 3 in the AT group and none in the AC group $(P=.24)$; for type 2,1 in the AT group and none in the AC group $(P=1.00)$; for type 3a, 1 in the AT group and 1 in the $\mathrm{AC}$ group $(P=1.00)$. Also, chest drainage volumes showed no significant difference between the 2 groups (Figure 4). No deaths, perioperative MI, or repeated revascularization occurred within 30 postoperative days. Length of hospital stay showed no significant difference between the AT and AC groups $(7.3 \pm 2.4$ days vs $7.2 \pm 2.2$ days, $P=.68)$.

\section{DISCUSSION}

The current study compared the pharmacokinetic characteristics and safety between ticagrelor and clopidogrel in patients receiving $\mathrm{CABG}$. The results showed that ticagrelor had a quicker onset and higher peak platelet inhibition rate; meanwhile, there was no significant difference in bleeding events between the ticagrelor and clopidogrel groups.

For patients receiving CABG, it is crucial to initiate antiplatelet therapy as early as possible after the procedure. Mangano ${ }^{15}$ found that the mortality was significantly lower in patients who started aspirin therapy within 48 hours after surgery than those in the control group $(1.3 \%$ vs $4.0 \%$, $P<.001)$. In fact, patients receiving CABG had abnormal activation of platelet function postoperatively, which persists until 30 days after the procedure. ${ }^{12}$ Accordingly, multiple studies further evaluated the efficacy of the AC DAPT regimen, but the results were inconsistent, which may be related to the long peak time of clopidogrel and significant individual difference in efficacy. ${ }^{6,16}$

Those limitations of clopidogrel make the timing of DAPT a concern for clinicians. As mentioned earlier, 30 days postoperation is a critical period for antiplatelet 
TABLE 2. Platelet function test at various time points

\begin{tabular}{|c|c|c|c|c|c|c|c|}
\hline & $\mathbf{0 ~ h}$ & $2 \mathrm{~h}$ & $8 \mathrm{~h}$ & $24 \mathrm{~h}$ & $72 \mathrm{~h}$ & $720 \mathrm{~h}$ & $P^{*}$ \\
\hline \multicolumn{7}{|c|}{ IPA measures } & $<.0001$ \\
\hline $\mathrm{AT}$ & - & $34.2(9.1-66.0)$ & $63.4(47.1-71.3)$ & $65.2(55.0-71.2)$ & $61.6(50.2-73.3)$ & $64.7(53.0-74.7)$ & \\
\hline $\mathrm{AC}$ & - & $5.3(-14.3-22.0)$ & $13.5(-2.9-28.5)$ & $21.2(-8.7-37.8)$ & $27.5(2.1-55.0)$ & $46.4(23.1-71.1)$ & \\
\hline$P^{* *}$ & - & $<.001$ & $<.001$ & $<.001$ & $<.001$ & .001 & \\
\hline \multicolumn{7}{|c|}{ PRI measures } & $<.001$ \\
\hline AT & $0.8(0.8-0.9)$ & $0.5(0.2-0.8)$ & $0.2(0.1-0.3)$ & $0.2(0.1-0.2)$ & $0.1(0.1-0.2)$ & $0.2(0.1-0.3)$ & \\
\hline $\mathrm{AC}$ & $0.8(0.8-0.9)$ & $0.8(0.7-09)$ & $0.8(0.8-0.9)$ & $0.8(0.7-0.9)$ & $0.5(0.2-0.7)$ & $0.3(0.1-0.6)$ & \\
\hline$P^{* *}$ & 1.0000 & $<.0001$ & $<.0001$ & $<.0001$ & $<.0001$ & .0210 & \\
\hline
\end{tabular}

Values are median (interquartile range). $P *$ indicates $P$ value of group-by-time interaction effect estimated by mixed-effects model. $P * *$ indicates $P$ value of Bonferroni multiplesignificance test correction. IPA, Inhibition of platelet aggregation; $A T$, aspirin + ticagrelor; $A C$, aspirin + clopidogrel; $P R I$, platelet reactivity index.

therapy because platelet functions are activated during this period. However, the slow onset may delay platelet inhibition and therefore negatively affect long-term prognosis. Ticagrelor may change this situation.

Unlike clopidogrel, ticagrelor is not a prodrug and its action does not rely on liver enzyme to convert it into an active drug, leading to significant improvement in bioavailability. ${ }^{10}$ The American Heart Association/American College of Cardiology guideline has included ticagrelor as the primary choice of antiplatelet drug for patients with acute coronary syndrome. ${ }^{3,17}$ But, it is yet to be determined whether the AT DAPT regimen can improve the efficacy of CABG. The PLATO subgroup study showed that the incidences of cardiac death and all-cause death were significantly lower in CABG recipients treated with ticagrelor than in the control group. ${ }^{18}$ Our study examined platelet functions after $\mathrm{CABG}$; the results showed that the mean IPA peak time was significantly faster in the AT group than in the AC group (235 vs 403 hours, $P=.0016$ ), and that the peak platelet inhibition rate in the AT group was approximately 1.5 times that in the AC group $(P<.001)$. This finding provided theoretical support for the subgroup study results of PLATO.

When compared with the nonsurgical population in the ONSET/OFFSET study, ${ }^{19}$ participants in the current study had shorter platelet inhibition and decreased IPAmax and PRImax. This difference was more notable in patients undergoing coronary artery bypass receiving clopidogrel compared with the nonsurgical population receiving clopidogrel, indicating that ticagrelor is more suitable for antiplatelet purpose after CABG. Although the mechanism underlying this difference is still unknown, we supposed

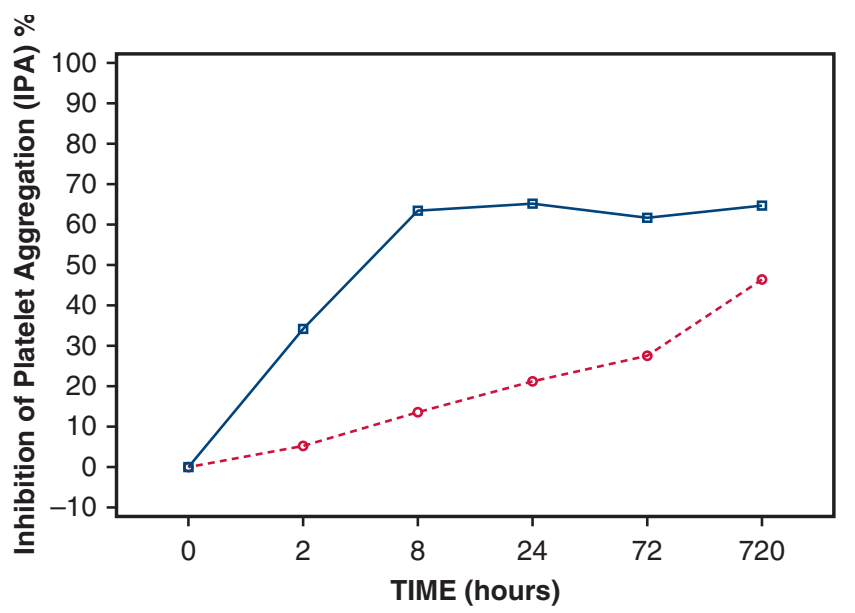

-.-- Aspirin+Clopidogrel (AC) - Aspirin+Ticagrelor (AT)

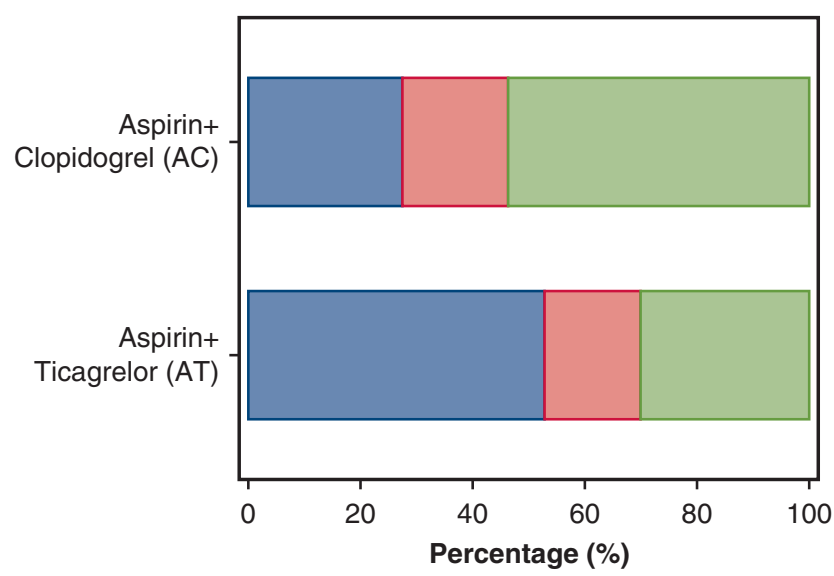

The time (h) to reach Maximum Inhibition of Platelet Aggregation (IPA)

$\square \leq 24 \mathrm{~h} \quad \square \geq 24 \mathrm{~h}, \leq 72 \mathrm{~h} \quad \square \geq 72 \mathrm{~h}, \leq 720 \mathrm{~h}$

\section{A}

FIGURE 2. IPA measured by light-transmittance aggregometry. A, IPA ( $\%$; ADP $20 \mu \mathrm{mol} / \mathrm{L}$; maximum extent) test at protocol time points. Data are expressed by median (interquartile range). IPA at 2 hours after the first drug administration was greater for AT than for AC. B, Distribution of the time to reach the IPAmax (\%; ADP $20 \mu \mathrm{mol} / \mathrm{L}$; maximum extent). Different colors represent the proportion of patients who reached the IPAmax in 3 time slots: within 24 hours, 24 to 72 hours, or 72 to 720 hours. More patients reached IPAmax within 24 hours in the AT group than in the AC group (52.9\% vs $27.5 \%$, $P=.002) . I P A$, Inhibition of platelet aggregation; $A C$, aspirin + clopidogrel; $A T$, aspirin + ticagrelor. 


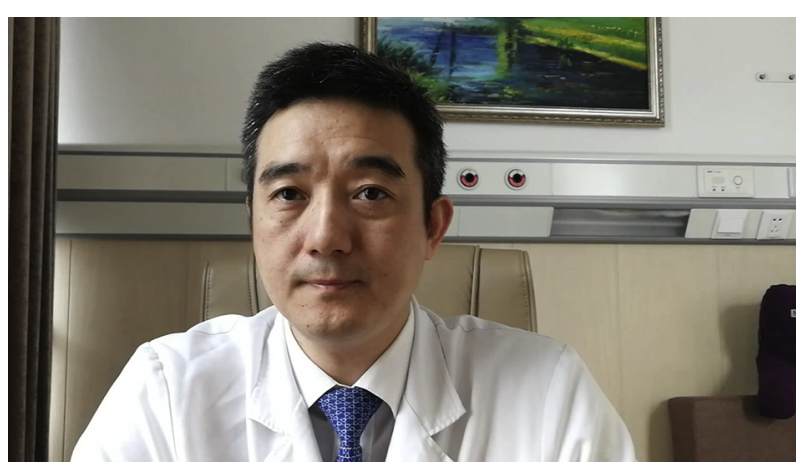

VIDEO 1. Dr Zhe Zheng introduces the main results and significance of the study. Video available at: https://www.jtcvs.org/article/S0022-5223 (18)32786-7/fulltext.

that this is related to an enhanced platelet activation in the surgical population.

The occurrence of postoperative bleeding events is another focus point in patients treated with ticagrelor after CABG. In a multicenter, randomized, controlled trial composed of 123 patients (stable angina), Gurbel and colleagues ${ }^{19}$ found that IPA declined significantly faster with ticagrelor than with clopidogrel after drug discontinuance, indicating that ticagrelor is safer in terms of bleeding events. However, our study observed no significant difference in chest drainage volume and total pleural fluid volume between the AT and AC groups 1 day after surgery. Similar to our study, the study by Saw and colleagues ${ }^{20}$ showed that no severe bleeding events (life-threatening hemorrhage or hemoglobin reduction $>3 \mathrm{~g} / \mathrm{dL}$ ) occurred within 3 months as a result of postoperative application of ticagrelor after CABG, but the incidence of mild bleeding events was 10 times higher in the ticagrelor group than in the placebo group. Despite the suggested safety of ticagrelor application, finding a balance between anticoagulation and bleeding is still an important direction for further studies.

\section{Study Limitations}

Because this study conducted only a 1-month follow-up, the short observation time may have caused the negative results in comparison of deaths, perioperative MI, and repeated revascularization. Also, we used CT to analyze 1-month vein graft patency and found negative results (Table E2). As described in the "Materials and Methods" section, the primary end point of this study was pharmacokinetic comparison. An expanded sample size and extended follow-up period are required. In the meantime, the proportion of clopidogrel resistance was unknown in this study because the clopidogrel gene was not analyzed, which may affect the study results.

Another limitation of the current study is that the data had relative low statistical power to evaluate the impact of cardiopulmonary bypass on platelet function. As shown in Tables E3 to E10, the platelet function was not statistically different between patients receiving on-pump

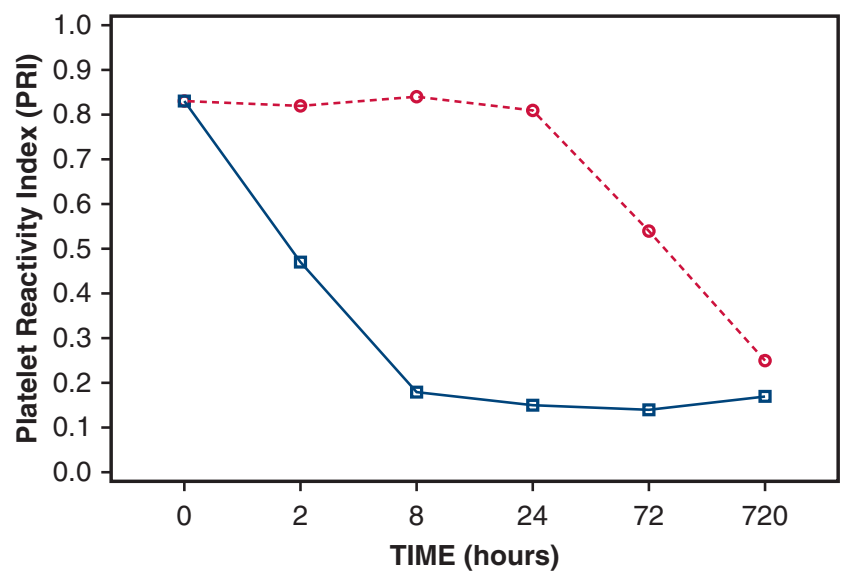

Aspirin+Clopidogrel (AC) — Aspirin+Ticagrelor (AT)

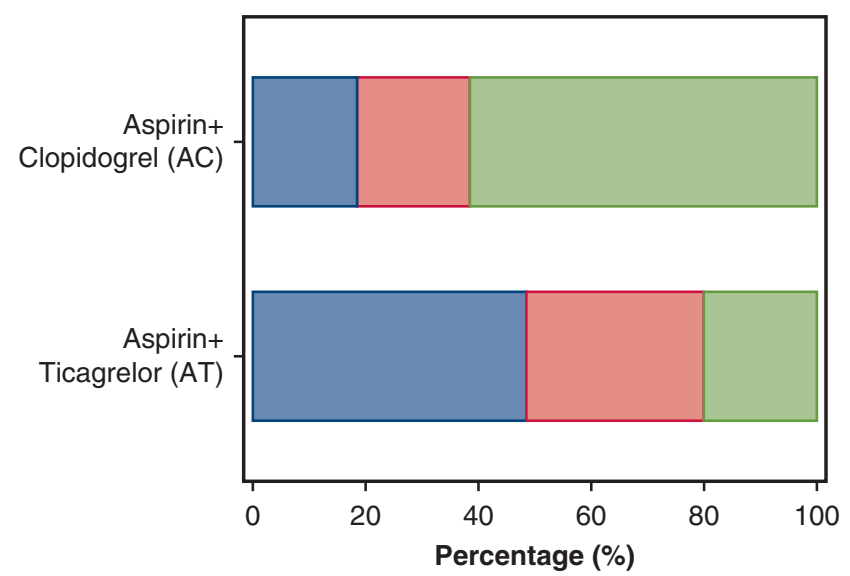

The time $(\mathrm{h})$ to reach minimum Platelet Reactivity Index (PRI)

$\leq 24 \mathrm{~h} \quad \square \geq 24 \mathrm{~h}, \leq 72 \mathrm{~h} \quad \square \geq 72 \mathrm{~h}, \leq 720 \mathrm{~h}$

A

B

FIGURE 3. PRI measured by vasodilator-stimulated phosphoprotein phosphorylation (VASP-P) levels. A, PRI measured by VASP-P levels at protocol time points. VASP-P levels tested by enzyme-linked immunosorbent assay. Data are expressed by median (interquartile range). More patients reached PRImin within 24 hours in the AT group than in the AC group (48.6\% vs $18.6 \%, P<.001)$. The slope from 0 to 24 hours was less in the AT group than in the AC group $(-0.019 \pm 0.001$ vs $-0.003 \pm 0.002 \mathrm{PRI} / \mathrm{h}, P<.001)$. B, Distribution of the time to reach PRImin. Different colors represent the proportion of patients who reached the PRImin in 3 time slots: within 24 hours, 24 to 72 hours, or 72 to 720 hours. PRI, Platelet reactivity index; $A C$, aspirin + clopidogrel; $A T$, aspirin + ticagrelor. 


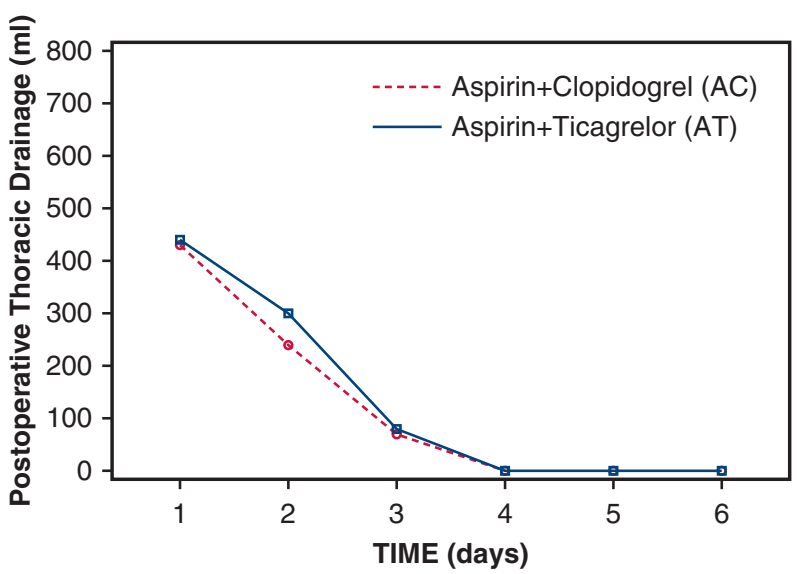

FIGURE 4. Postoperative thoracic drainage. Data are expressed by mean \pm standard error of the mean. AT versus AC, $P=.771$. Chest drainage volumes were not significantly different in the 2 groups. $A C$, Aspirin + clopidogrel; $A T$, aspirin + ticagrelor.

CABG and patients receiving off-pump CABG. In the subgroup analysis in on-pump or off-pump patients, the onset of action was faster and the peak IPA was higher with AT than with AC.

\section{CONCLUSIONS}

In patients receiving $\mathrm{CABG}$, the onset of action was faster and the peak IPA was higher with ticagrelor than with clopidogrel, whereas the medication safety within 1 month after surgery was similar between the 2 drugs.

\section{Conflict of Interest Statement}

F.X. and Z.Z. received research grants from AstraZeneca $\mathrm{Co}$, Ltd. All other authors have nothing to disclose with regard to commercial support.

The authors thank Jing Li, MD (China Oxford Centre for International Health Research, Fuwai Hospital Chinese Academy of Medical Sciences, Beijing, China), for editorial assistance.

\section{References}

1. Fitzgibbon GM, Kafka HP, Leach AJ, Keon WJ, Hooper GD, Burton JR. Coronary bypass graft fate and patient outcome: angiographic follow-up of 5,065 grafts related to survival and reoperation in 1,388 patients during 25 years. $J$ Am Coll Cardiol. 1996;28:616-26.

2. Motwani JG, Topol EJ. Aortocoronary saphenous vein graft disease: pathogenesis, predisposition, and prevention. Circulation. 1998:97:916-31.

3. Levine GN, Bates ER, Bittl JA, Brindis RG, Fihn SD, Fleisher LA, et al. 2016 ACC/AHA guideline focused update on duration of dual antiplatelet therapy in patients with coronary artery disease: A report of the American College of Cardiology/American Heart Association task force on clinical practice guidelines: an update of the 2011 ACCF/AHA/SCAI guideline for percutaneous coronary intervention, $2011 \mathrm{ACCF} / \mathrm{AHA}$ guideline for coronary artery bypass graft surgery, 2012 ACC/AHA/ACP/AATS/PCNA/SCAI/STS guideline for the diagnosis and management of patients with stable ischemic heart disease, 2013 ACCF/AHA guideline for the management of ST-elevation myocardial infarction, 2014 AHA/ACC guideline for the management of patients with non-ST-elevation acute coronary syndromes, and 2014 ACC/AHA guideline on perioperative cardiovascular evaluation and management of patients undergoing noncardiac surgery. Circulation. 2016;134:e123-55.

4. Yanagawa B, Ruel M, Bonneau C, Lee MM, Chung J, Al Shouli S, et al. Dual antiplatelet therapy used by Canadian cardiac surgeons. J Thorac Cardiovasc Surg. 2015;150:1548-54.

5. Deo SV, Dunlay SM, Park SJ. Dual antiplatelet therapy after coronary artery bypass grafting: does off/on-pump play a role? Am J Cardiol. 2014;113: 1085

6. Gao G, Zheng Z, Pi Y, Lu B, Lu J, Hu S. Aspirin plus clopidogrel therapy increases early venous graft patency after coronary artery bypass surgery a single-center, randomized, controlled trial. J Am Coll Cardiol. 2010;56:1639-43.

7. Nocerino AG, Achenbach S, Taylor AJ. Meta-analysis of effect of single versus dual antiplatelet therapy on early patency of bypass conduits after coronary artery bypass grafting. Am J Cardiol. 2013;112:1576-9.

8. Gurbel PA, Bliden KP, Saucedo JF, Suarez TA, DiChiara J, Antonino MJ, et al. Bivalirudin and clopidogrel with and without eptifibatide for elective stenting: effects on platelet function, thromboelastographic indexes, and their relation to periprocedural infarction results of the CLEAR PLATELETS-2 (clopidogre with eptifibatide to arrest the reactivity of platelets) study. J Am Coll Cardiol. 2009;53:648-57.

9. Gurbel PA, Bliden KP, Zaman KA, Yoho JA, Hayes KM, Tantry US. Clopidogrel loading with eptifibatide to arrest the reactivity of platelets: results of the clopidogrel loading with eptifibatide to arrest the reactivity of platelets (CLEAR PLATELETS) study. Circulation. 2005;111:1153-9.

10. Husted S, Emanuelsson H, Heptinstall S, Sandset PM, Wickens M, Peters G Pharmacodynamics, pharmacokinetics, and safety of the oral reversible P2Y12 antagonist AZD6140 with aspirin in patients with atherosclerosis: a doubleblind comparison to clopidogrel with aspirin. Eur Heart J. 2006;27:1038-47.

11. Parolari A, Mussoni L, Frigerio M, Naliato M, Alamanni F, Galanti A, et al Increased prothrombotic state lasting as long as one month after on-pump and off-pump coronary surgery. J Thorac Cardiovasc Surg. 2005;130:303-8.

12. Van Poucke S, Stevens K, Wetzels R, Kicken C, Verhezen P, Theunissen M, et al Early platelet recovery following cardiac surgery with cardiopulmonary bypass Platelets. 2016;27:751-7.

13. Hu S, Zheng Z, Yuan X, Wang W, Song Y, Sun H, et al. Increasing longterm major vascular events and resource consumption in patients receiving off-pump coronary artery bypass: a single-center prospective observational study. Circulation. 2010;121:1800-8.

14. Mehran R, Rao SV, Bhatt DL, Gibson CM, Caixeta A, Eikelboom J, et al. Standardized bleeding definitions for cardiovascular clinical trials: a consensus report from the bleeding academic research consortium. Circulation. 2011;123: 2736-47.

15. Mangano DT. Aspirin and mortality from coronary bypass surgery. $N$ Engl J Med 2002;347:1309-17.

16. Kulik A, Le May MR, Voisine P, Tardif JC, Delarochelliere R, Naidoo S, et al. Aspirin plus clopidogrel versus aspirin alone after coronary artery bypass graft ing: the clopidogrel after surgery for coronary artery disease (CASCADE) Trial. Circulation. 2010;122:2680-7.

17. Kulik A, Ruel M, Jneid H, Ferguson TB, Hiratzka LF, Ikonomidis JS, et al. Secondary prevention after coronary artery bypass graft surgery: a scientific statement from the American Heart Association. Circulation. 2015;131:927-64.

18. Brilakis ES, Held C, Meier B, Cools F, Claeys MJ, Cornel JH, et al. Effect of ticagrelor on the outcomes of patients with prior coronary artery bypass graft surgery: insights from the Platelet inhibition and patient outcomes (PLATO) trial. Am Heart J. 2013;166:474-80.

19. Gurbel PA, Bliden KP, Butler K, Tantry US, Gesheff T, Wei C, et al. Randomized double-blind assessment of the ONSET and OFFSET of the antiplatelet effects of ticagrelor versus clopidogrel in patients with stable coronary artery disease: the ONSET/OFFSET study. Circulation. 2009;120:2577-85.

20. Saw J, Wong GC, Mayo J, Bernstein V, Mancini GB, Ye J, et al. Ticagrelor and aspirin for the prevention of cardiovascular events after coronary artery bypass graft surgery. Heart. 2016;102:763-9.

Key Words: CABG, dual antiplatelet therapy, platelet function, postoperation 
TABLE E1. Standardized bleeding definitions for Bleeding Academic Research Consortium

Type 0:

No bleeding

Type 1:

Bleeding that is not actionable and does not cause the patient to seek unscheduled performance of studies, hospitalization, or treatment by a healthcare professional; may include episodes leading to self-discontinuation of medical therapy by the patient without consulting a healthcare professional.

Type 2:

Any overt, actionable sign of hemorrhage (eg, more bleeding than would be expected for a clinical circumstance, including bleeding found by imaging alone) that does not fit the criteria for type 3, 4, or 5 but does meet at least 1 of the following criteria:

(1) requiring nonsurgical, medical intervention by a healthcare professional, (2) leading to hospitalization or increased level of care, or (3) prompting evaluation.

Type 3

Type $3 a$

Overt bleeding plus hemoglobin decrease of 3 to $5 \mathrm{~g} / \mathrm{dL} *$ (provided hemoglobin decrease is related to bleed); any transfusion with overt bleeding. Type $3 b$

Overt bleeding plus hemoglobin decrease $5 \mathrm{~g} / \mathrm{dL} *$ (provided hemoglobin decrease is related to bleed); cardiac tamponade; bleeding requiring surgical intervention for control (excluding dental/nasal/skin/hemorrhoid); bleeding requiring intravenous vasoactive agents.

Type 3c

Intracranial hemorrhage (does not include microbleeds or hemorrhagic transformation, does include intraspinal); subcategories confirmed by autopsy or imaging or lumbar puncture; intraocular bleed compromising vision.

Type 4:

CABG-related bleeding; perioperative intracranial bleeding within $48 \mathrm{~h}$; reoperation after closure of sternotomy for the purpose of controlling bleeding; transfusion of $5 \mathrm{U}$ whole blood or packed red blood cells within a 48 -h period; chest tube output $2 \mathrm{~L}$ within a 24 -h period

Type 5:

Fatal bleeding.

Type 5 a

Probable fatal bleeding; no autopsy or imaging confirmation but clinically suspicious

Type $5 b$

Definite fatal bleeding; overt bleeding or autopsy or imaging confirmation.

Mehran R, Rao SV, Bhatt DL, Gibson CM, Caixeta A, Eikelboom J, et al. Standardized bleeding definitions for cardiovascular clinical trials: a consensus report from the Bleeding Academic Research Consortium. Circulation. 2011;123:2742, Table 3. CABG, Coronary artery bypass grafting.

TABLE E2. Computed tomography examination of venous graft patency rate

\begin{tabular}{lllll}
\hline & Total $(\mathbf{N}=\mathbf{3 5 7})$ & AC $(\mathbf{N}=\mathbf{1 8 1})$ & AT $(\mathbf{N}=\mathbf{1 7 6})$ & $\boldsymbol{P}$ value \\
\hline Vein graft failure $(\%, \mathrm{n})$ & $8.7 \%, 31$ & $11.6 \%, 21$ & $5.7 \%, 10$ & .0470 \\
Vein graft occlusion $(\%, \mathrm{n})$ & $3.9 \%, 14$ & $3.9 \%, 7$ & $4 \%, 7$ & .9574
\end{tabular}

The time of CT examination is 1 month after surgery. The vein graft failure refers to the presence of bridge vessel stenosis greater than $50 \%$ observed by CT. $A C$, Aspirin + clopidogrel; $A T$, aspirin + ticagrelor. 
TABLE E3. Patient characteristics of groups by on/off pump

\begin{tabular}{|c|c|c|c|c|}
\hline Variable & Overall $(n=140)$ & On pump $(n=26)$ & Off pump $(n=114)$ & $P$ \\
\hline Age $(y)($ mean \pm SD $)$ & $59.5 \pm 8.2$ & $58.1 \pm 8.2$ & $59.8 \pm 8.2$ & .4511 \\
\hline Gender (female) & $25 \%(35)$ & $7.7 \%(2)$ & $28.9 \%(33)$ & .0239 \\
\hline Weight & $72.3 \pm 10.5$ & $74.6 \pm 12$ & $71.8 \pm 10.1$ & .2256 \\
\hline Hypertension & $65.7 \%(92)$ & $61.5 \%(16)$ & $66.7 \%(76)$ & .6191 \\
\hline Diabetes mellitus & $32.9 \%(46)$ & $23.1 \%(6)$ & $35.1 \%(40)$ & .2394 \\
\hline Previous AMI & $37.9 \%(53)$ & $42.3 \%(11)$ & $36.8 \%(42)$ & .6041 \\
\hline $\mathrm{EF}(\%)$ & $58.9 \pm 9.6$ & $60.6 \pm 8.8$ & $58.5 \pm 9.8$ & .2806 \\
\hline LVEDD (mm) & $49.8 \pm 5.8$ & $50.2 \pm 3.9$ & $49.7 \pm 6.1$ & .6598 \\
\hline $\begin{array}{c}\text { CCS class } \\
1 \\
2 \\
3 \\
4\end{array}$ & $\begin{array}{l}10.7 \%(15) \\
44.3 \%(62) \\
27.9 \%(39) \\
17.1 \%(24)\end{array}$ & $\begin{array}{l}7.7 \%(2) \\
53.8 \%(14) \\
26.9 \%(7) \\
11.5 \%(3)\end{array}$ & $\begin{array}{l}11.4 \%(13) \\
42.1 \%(48) \\
28.1 \%(32) \\
18.4 \%(21)\end{array}$ & .6762 \\
\hline $\begin{array}{l}\text { NYHA } \\
\text { I (\%) } \\
\text { II (\%) } \\
\text { III }(\%) \\
\text { IV }(\%)\end{array}$ & $\begin{array}{c}10 \%(14) \\
71.4 \%(100) \\
16.4 \%(23) \\
2.1 \%(3)\end{array}$ & $\begin{array}{c}7.7 \%(2) \\
84.6 \%(22) \\
7.7 \%(2) \\
0 \%(0)\end{array}$ & $\begin{array}{c}10.5 \%(12) \\
68.4 \%(78) \\
18.4 \%(21) \\
2.6 \%(3)\end{array}$ & .3742 \\
\hline Hemoglobin (g/L) & $134.7 \pm 17$ & $140.2 \pm 12.8$ & $133.5 \pm 17.6$ & .0599 \\
\hline Platelet $(* 109 / \mathrm{L})$ & $223.3 \pm 61.9$ & $216 \pm 61.1$ & $225 \pm 62.3$ & .4262 \\
\hline Prothrombin time (s) & $14.5 \pm 13.3$ & $17.4 \pm 22$ & $13.9 \pm 10.4$ & .1895 \\
\hline Creatinine (mmol/L) Preoperative medication & $83.2 \pm 16.1$ & $86.6 \pm 11.7$ & $82.4 \pm 16.8$ & .0465 \\
\hline Beta-blocker & $100 \%(140)$ & $100 \%(26)$ & $100 \%(114)$ & \\
\hline ACEI/ARB & $31.4 \%(44)$ & $30.8 \%(8)$ & $31.6 \%(36)$ & .9360 \\
\hline Calcium antagonist & $45 \%(63)$ & $38.5 \%(10)$ & $46.5 \%(53)$ & .4577 \\
\hline Statins & $70.7 \%(99)$ & $76.9 \%(20)$ & $69.3 \%(79)$ & .4407 \\
\hline Nitrates & $92.9 \%(130)$ & $92.3 \%(24)$ & $93 \%(106)$ & .9040 \\
\hline euroSCORE II & $1.2 \pm 0.5$ & $1.2 \pm 0.7$ & $1.2 \pm 0.5$ & .1418 \\
\hline STS score & $0.7 \pm 0.6$ & $0.6 \pm 0.3$ & $0.8 \pm 0.6$ & .1804 \\
\hline Treatment group (AT) & $50 \%(70)$ & $46.2 \%(12)$ & $50.9 \%(58)$ & .6638 \\
\hline \multicolumn{5}{|c|}{ Surgical data } \\
\hline Variable & Overall $(n=140)$ & On pump $(n=26)$ & Off pump $(n=114)$ & $P$ \\
\hline No. of grafts & & & & .8809 \\
\hline 1 & $0.7 \%$ & $0 \%(0)$ & $0.9 \%$ & \\
\hline 2 & $7.9 \%$ & $7.7 \%$ & $7.9 \%$ & \\
\hline 3 & $30.7 \%(43)$ & $38.5 \%(10)$ & $28.9 \%$ & \\
\hline 4 & $52.1 \%(73)$ & $50 \%$ & $52.6 \%(60)$ & \\
\hline 5 & $7.9 \%$ & $3.8 \%$ & $8.8 \%(10)$ & \\
\hline 6 & $0.7 \%(1)$ & $0 \%(0)$ & $0.9 \%$ & \\
\hline
\end{tabular}

$\overline{S D}$, Standard deviation; $A M I$, acute myocardial infarction; $E F$, ejection fraction; $L V E D D$, left ventricular end-diastolic diameter; $C C S$, Canadian Cardiovascular Society; $N Y H A$, New York Heart Association; $A C E I / A R B$, angiotensin-converting enzyme inhibitor/angiotensin receptor blocker; euroSCORE, European System for Cardiac Operative Risk Evaluation; STS, Society of Thoracic Surgeons; $A T$, aspirin + ticagrelor. 
TABLE E4. Platelet function test at various time points of groups by on/off pump

\begin{tabular}{|c|c|c|c|c|c|c|c|}
\hline & $\mathbf{0 ~ h}$ & $2 \mathrm{~h}$ & $8 \mathrm{~h}$ & $24 \mathrm{~h}$ & $72 \mathrm{~h}$ & $720 \mathrm{~h}$ & $P^{*}$ \\
\hline IPA measures & & & & & & & $<.001$ \\
\hline On pump & - & $18.3(-9.2-30.6)$ & $30(3.7-57.8)$ & $53.2(15.7-68.9)$ & $47.7(17.5-73)$ & $69.9(41.4-78.9)$ & \\
\hline Off pump & - & $20.6(1.4-48.7)$ & $46.6(11.7-65.2)$ & $49.6(17.5-67.1)$ & $53.5(26.3-68.9)$ & $57.3(39.9-71.1)$ & \\
\hline$P^{* *}$ & - & 1.0000 & 1.0000 & 1.0000 & 1.0000 & 1.0000 & \\
\hline PRI measures & & & & & & & $<.001$ \\
\hline On pump & $0.9(0.8-0.9)$ & $0.8(0.4-0.9)$ & $0.8(0.2-0.9)$ & $0.6(0.2-0.8)$ & $0.2(0.1-0.5)$ & $0.3(0.1-0.4)$ & \\
\hline Off pump & $0.8(0.8-0.9)$ & $0.7(0.4-0.8)$ & $0.4(0.2-0.8)$ & $0.3(0.1-0.8)$ & $0.2(0.1-0.6)$ & $0.2(0.1-0.3)$ & \\
\hline$P^{* *}$ & 1.0000 & 1.0000 & 1.0000 & 1.0000 & 1.0000 & 1.0000 & \\
\hline
\end{tabular}

Values are median (25th-75th percentile). $P^{*}$ indicates $P$ value of group-by-time interaction effect estimated by mixed-effects model. $P^{* *}$ indicates $P$ value of Bonferroni multiple-significance test correction. IPA, Inhibition of platelet aggregation; PRI, platelet reactivity index

TABLE E5. Baseline characteristics of off-pump subgroup

\begin{tabular}{|c|c|c|c|c|}
\hline Variable & Overall $(n=114)$ & $\mathbf{A T}(\mathbf{n}=58)$ & $\mathrm{AC}(\mathrm{n}=56)$ & $P$ value \\
\hline $\operatorname{Age}(y)($ mean $\pm \mathrm{SD})$ & $59.8 \pm 8.2$ & $58.7 \pm 9.1$ & $60.9 \pm 7.1$ & .3085 \\
\hline Weight & $71.8 \pm 10.1$ & $73 \pm 10.4$ & $70.4 \pm 9.7$ & .3060 \\
\hline $\mathrm{EF}(\%)$ & $58.5 \pm 9.8$ & $58.2 \pm 10.8$ & $58.8 \pm 8.6$ & .9706 \\
\hline LVEDD (mm) & $49.7 \pm 6.1$ & $50.3 \pm 6.7$ & $49.1 \pm 5.5$ & .6619 \\
\hline Hemoglobin $(\mathrm{g} / \mathrm{L})$ & $133.5 \pm 17.6$ & $135.9 \pm 13.9$ & $131 \pm 20.5$ & .1990 \\
\hline Prothrombin time (s) & $13.9 \pm 10.4$ & $14.8 \pm 14.6$ & $12.9 \pm 0.6$ & .9638 \\
\hline Creatinine $(\mathrm{mmol} / \mathrm{L})$ & $82.4 \pm 16.8$ & $82.7 \pm 16.3$ & $82 \pm 17.5$ & .5903 \\
\hline euroSCORE II & $1.2 \pm 0.4$ & $1.1 \pm 0.4$ & $1.2 \pm 0.4$ & .5536 \\
\hline STS score & $0.8 \pm 0.6$ & $0.7 \pm 0.6$ & $0.8 \pm 0.6$ & .2208 \\
\hline
\end{tabular}

$A T$, Aspirin + ticagrelor; $A C$, aspirin + clopidogrel; $S D$, standard deviation; $E F$, ejection fraction; $L V E D D$, left ventricular end-diastolic dimension; euroSCORE, European System for Cardiac Operative Risk Evaluation; STS, Society of Thoracic Surgeons.

TABLE E6. Inhibition of platelet aggregation of off-pump subgroup

\begin{tabular}{|c|c|c|c|c|}
\hline Variable & Overall $(n=114)$ & $\mathbf{A T}(\mathbf{n}=58)$ & $\mathrm{AC}(\mathrm{n}=56)$ & $P$ value \\
\hline ipa_adp0-2 & $22.5 \pm 35.1$ & $36.6 \pm 34.4$ & $7.1 \pm 28.9$ & $<.0001$ \\
\hline ipa_adp0-8 & $38.3 \pm 34.1$ & $57.6 \pm 24.1$ & $16.8 \pm 30.7$ & $<.0001$ \\
\hline ipa_adp0-24 & $40.3 \pm 34.2$ & $61.4 \pm 20$ & $16.7 \pm 31.3$ & $<.0001$ \\
\hline ipa_adp0-72 & $43.1 \pm 32.3$ & $58 \pm 20.2$ & $26.8 \pm 35.2$ & $<.0001$ \\
\hline ipa_adp0-720 & $50.9 \pm 32.6$ & $59.8 \pm 27.8$ & $40.7 \pm 35$ & .0020 \\
\hline
\end{tabular}

$P$ value is used multiple test by Bonferroni correction. $A T$, Aspirin + ticagrelor; $A C$, aspirin + clopidogrel. 
TABLE E7. Platelet reactivity index of off-pump subgroup

\begin{tabular}{lcccc}
\hline Variable & Overall $(\mathbf{n}=\mathbf{1 1 4})$ & AT $(\mathbf{n}=\mathbf{5 8})$ & AC $(\mathbf{n}=\mathbf{5 6})$ & $\boldsymbol{P}$ value \\
\hline pri1 & $0.8 \pm 0.1$ & $0.8 \pm 0.1$ & $0.8 \pm 0.2$ & 1.0000 \\
pri2 & $0.6 \pm 0.3$ & $0.5 \pm 0.3$ & $0.8 \pm 0.2$ & $<.0001$ \\
pri3 & $0.5 \pm 0.3$ & $0.3 \pm 0.2$ & $0.8 \pm 0.2$ & $<.0001$ \\
pri4 & $0.4 \pm 0.3$ & $0.2 \pm 0.1$ & $0.7 \pm 0.2$ & $<.0001$ \\
pri5 & $0.3 \pm 0.3$ & $0.1 \pm 0.1$ & $0.5 \pm 0.3$ & $<.0001$ \\
pri6 & $0.3 \pm 0.2$ & $0.2 \pm 0.2$ & $0.3 \pm 0.3$ & .0222 \\
\hline
\end{tabular}

$P$ value is used multiple test by Bonferroni correction. $A T$, Aspirin + ticagrelor; $A C$, aspirin + clopidogrel.

TABLE E8. Baseline characteristics of on-pump subgroup

\begin{tabular}{|c|c|c|c|c|}
\hline Variable & Overall $(n=26)$ & $\mathbf{A T}(\mathrm{n}=12)$ & $\mathrm{AC}(\mathrm{n}=14)$ & $P$ value \\
\hline Age $(y)($ mean \pm SD) & $58.1 \pm 8.2$ & $58.3 \pm 8.2$ & $58 \pm 8.5$ & .9795 \\
\hline Weight & $74.6 \pm 12$ & $71.8 \pm 14.7$ & $77.1 \pm 8.9$ & .3030 \\
\hline $\mathrm{EF}(\%)$ & $60.6 \pm 8.8$ & $59.1 \pm 9.6$ & $61.9 \pm 8.3$ & .4094 \\
\hline LVEDD (mm) & $50.2 \pm 3.9$ & $49.3 \pm 3.6$ & $50.9 \pm 4.2$ & .3509 \\
\hline Hemoglobin (g/L) & $140.2 \pm 12.8$ & $141.7 \pm 16.8$ & $139 \pm 8.5$ & .8569 \\
\hline Prothrombin time (s) & $17.4 \pm 22$ & $12.9 \pm 0.7$ & $21.2 \pm 29.9$ & .2069 \\
\hline Creatinine (mmol/L) & $86.6 \pm 11.7$ & $81.8 \pm 11.3$ & $90.8 \pm 10.8$ & .0760 \\
\hline euroSCORE II & $1.1 \pm 0.5$ & $1.2 \pm 0.5$ & $1.2 \pm 0.4$ & .6578 \\
\hline STS score & $0.6 \pm 0.3$ & $0.7 \pm 0.5$ & $0.5 \pm 0.2$ & .3961 \\
\hline
\end{tabular}

$A T$, Aspirin + ticagrelor; $A C$, aspirin + clopidogrel; $S D$, standard deviation; $E F$, ejection fraction; $L V E D D$, left ventricular end-diastolic dimension; euroSCORE, European System for Cardiac Operative Risk Evaluation; STS, Society of Thoracic Surgeons.

TABLE E9. Inhibition of platelet aggregation of on-pump subgroup

\begin{tabular}{lcccc}
\hline \multicolumn{1}{c}{ Variable } & Overall $(\mathbf{n}=\mathbf{2 6})$ & AT $(\mathbf{n}=\mathbf{1 2})$ & AC $(\mathbf{n}=\mathbf{1 4})$ & $\boldsymbol{P}$ value \\
\hline ipa_adp0-2 & $11.3 \pm 38.8$ & $41.2 \pm 24.3$ & $-12.2 \pm 31.2$ & .0015 \\
ipa_adp0-8 & $26.9 \pm 43.6$ & $57.5 \pm 21.5$ & $-1.4 \pm 39.6$ & .0015 \\
ipa_adp0-24 & $39.5 \pm 45.1$ & $69.8 \pm 15.9$ & $13.5 \pm 46$ & .0020 \\
ipa_adp0-72 & $44.6 \pm 32.7$ & $65 \pm 19.9$ & $27.1 \pm 31.9$ & .0155 \\
ipa_adp0-720 & $56.6 \pm 34.2$ & $58.9 \pm 32.8$ & $54.7 \pm 36.6$ & 1.0000 \\
\hline
\end{tabular}

$P$ value is used multiple test by Bonferroni correction. $A T$, Aspirin + ticagrelor; $A C$, aspirin + clopidogrel.

TABLE E10. Platelet reactivity index of on-pump subgroup

\begin{tabular}{lcccr}
\hline Variable & Overall $(\mathbf{n}=\mathbf{2 6})$ & AT $(\mathbf{n}=\mathbf{1 2})$ & AC $(\mathbf{n}=\mathbf{1 4})$ & \multicolumn{1}{c}{$\boldsymbol{P}$ value } \\
\hline pri1 & $0.8 \pm 0.1$ & $0.8 \pm 0.1$ & $0.8 \pm 0.1$ & $0.8 \pm 0.1$ \\
pri2 & $0.7 \pm 0.3$ & $0.5 \pm 0.3$ & $0.8 \pm 0.1$ & .1476 \\
pri3 & $0.6 \pm 0.3$ & $0.3 \pm 0.3$ & $0.8 \pm 0.1$ & .0012 \\
pri4 & $0.5 \pm 0.3$ & $0.2 \pm 0.1$ & $0.5 \pm 0.2$ & $<.0001$ \\
pri5 & $0.3 \pm 0.3$ & $0.1 \pm 0.1$ & $0.4 \pm 0.3$ & .0036 \\
pri6 & $0.3 \pm 0.2$ & $0.2 \pm 0.1$ & 1.0000 \\
\hline
\end{tabular}

$P$ value is used multiple test by Bonferroni correction. $A T$, Aspirin + ticagrelor; $A C$, aspirin + clopidogrel. 